\title{
Addendum: Single Cluster Monte Carlo Dynamics for the Ising Model $^{1}$
}

\author{
P. Tamayo, ${ }^{2}$ R. C. Brower, ${ }^{3}$ and W. Klein ${ }^{2}$
}

Received March 29, 1990

After the manuscript was accepted, we learned that Wolff ${ }^{(1)}$ had independently confirmed our conclusion that the Wolff algorithm relaxes faster than the Swendsen-Wang algorithm. On the other hand, Heermann and Burkitt $^{(2)}$ in two dimensions and Wang ${ }^{(3)}$ in three dimensions found more accurate exponents $z$ for the Swendsen-Wang algorithm which are much lower than previously published. These new exponents at first seem to invalidate our conclusion that the Wolf algorithm relaxes faster, but that conclusion remains valid nevertheless. For example, for $L=64$, the Wolff algorithm has a relaxation time of about 2.4, whereas the Swendsen-Wang algorithm relaxes in about 4.6 time steps. According to Wang, ${ }^{(4)}$ this advantage also carries over to the dilute Ising model in three dimensions.

\section{REFERENCES}

1. U. Wolff, preprint, Universitat Kiel.

2. D. W. Heermann and A. N. Burkitt, Physica A 162:210 (1990).

3. J. S. Wang, Physica A, preprint.

4. J. S. Wang, private communication.

\footnotetext{
${ }^{1}$ This work appeared in J. Stat. Phys. 58:1083 (1990).

${ }^{2}$ Physics Department and Center for Polymer Studies, Boston University, Boston, Massachusetts.

${ }^{3}$ Department of Electrical, Computer and Systems Engineering, and Physics Department, Boston University, Boston, Massachusetts.
} 